\title{
Hemicrania Continua Associated with Classic Scintillating Scotoma
}

\author{
Eva Auffenberg $^{\mathrm{a}} \quad$ Friedemann Bender $^{\mathrm{b}} \quad$ Tobias Freilinger $^{\mathrm{a}}$ \\ ${ }^{a}$ Department of Neurology and Epileptology, Hertie-Institute for Clinical Brain Research \\ $(\mathrm{HIH})$, Tübingen, Germany; ${ }^{b}$ Department of Neurodegeneration, Hertie-Institute for \\ Clinical Brain Research (HIH), Tübingen, Germany
}

\section{Keywords}

Hemicrania continua $\cdot$ Aura Visual aura $\cdot$ Scintillating scotoma $\cdot$ Trigeminal autonomic cephalalgia

\begin{abstract}
Hemicrania continua $(\mathrm{HC})$ is a rare primary headache disorder, characterized by persistent unilateral pain associated with cranial autonomic symptoms and prompt response to indomethacin. While migrainous features (including aura) have been recognized in cluster headache, there have been only single reports of HC with aura. Here, we report the case of a 53year-old man with constant right-sided headache and superimposed exacerbations to severe pain lasting for several hours. Secondary etiologies were excluded, and a diagnosis of $\mathrm{HC}$ was established after prompt and complete response to treatment with indomethacin. During an episode of pain exacerbation, for the first time the patient experienced an episode of transient visual disturbances compatible with scintillating scotoma. We propose a potential link between $\mathrm{HC}$ and visual aura, which parallels similar observations in other trigeminal autonomic cephalalgias and more specifically confirms previous observational data on aura in $\mathrm{HC}$, thus highlighting potentially shared pathophysiological mechanisms.
\end{abstract}




\section{Introduction}

Hemicrania continua (HC) is a rare primary headache disorder, which, according to the International Classification of Headache Disorders (ICHD)-III $\beta$ [1], is part of the trigeminal autonomic cephalalgias (TACs).

Since its initial description in 1984, over 100 cases have been reported [2]. It is characterized by strictly unilateral, continuous pain with moderate severity and episodic exacerbations to severe intensity lasting for hours, with prompt response to treatment with indomethacin as a hallmark feature. During pain exacerbations, patients suffer from additional ipsilateral trigeminal autonomic symptoms, such as conjunctival injection or lacrimation, nasal congestion or rhinorrhea, ptosis, and/or miosis.

While migrainous features have been recognized as part of HC [2-4], there have been only single reports of $\mathrm{HC}$ associated with aura symptoms [5]. Here, we report clinical details of a 53-year-old previously healthy man with co-occurrence of $\mathrm{HC}$ and classical scintillating scotoma, discuss pathophysiological implications, and review the available literature on the topic.

\section{Case Presentation}

We report a previously healthy 53-year-old Caucasian male who presented to our outpatient headache unit as an emergency. Past medical history was remarkable for surgery of the cervical spine several years ago as well as a diagnosis of Ménière disease, with a stable clinical course over the last few years but with residual sensorineural amblyacousia of the left ear. There was no daily medication; in particular, there was no current or previous history of overuse of analgesics. The patient was a heavy smoker (60 pack-years). There was no history of primary headache disorders, specifically no migraine. The patient reported a 4month history of constant unremitting pain in the right periorbital region. Pain intensity was described as low to medium (at least $2-3 / 10$ on the subjective analogue scale), with exacerbations to severe pain (i.e., 10/10) lasting for several hours. During exacerbations, additional right-sided symptoms (reddening of the eye, drooping of the eyelid, nasal congestion, and allodynia in the $\mathrm{V}_{1}$ innervated area) were noted. The precise frequency of exacerbations could not be assessed retrospectively, and no specific triggering factors during exacerbations could be established. Further, exacerbations occurred without nausea, vomiting, and photoor phonophobia, and there was no aggravation by exercise.

On his way to the hospital, the patient for the first time experienced binocular visual disturbances slowly spreading peripherally, comprising positive (i.e., scintillations) as well as negative (i.e., scotoma) features, with a total duration of 20-30 min; these symptoms coincided with a period of pain exacerbation.

The evaluation by an ENT specialist at the beginning of his complaints had not been conclusive, and a short course of oral antibiotics (precise substance unknown) led to only transient relief. Prior to the presentation to our hospital, the patient had been seen by another neurologist. Magnetic resonance imaging (MRI) of the brain (including the orbital structures and the cavernous sinus) had revealed no abnormalities, and Doppler sonography of extracranial arteries had been normal; in particular, extraocular muscles were found to be intact in all directions of gaze. Treatment with oral prednisolone (which was started assuming a cavernous sinus syndrome and tapered to $50 \mathrm{mg}$ q.d. at the day of admission) as well as oral nonsteroidal anti-inflammatory drugs had been without any effect on his complaints. 
On admission, neurological status revealed right-sided Horner syndrome and conjunctival injection (while the patient suffered from a pain exacerbation) but was otherwise normal. The patient was obese (body mass index of 32). Laboratory and cerebrospinal fluid analysis were unremarkable. Repeated cranial MRI revealed no pathology, in particular no orbital or cavernous sinus lesions, and MR angiography ruled out cervical artery dissection. Ophthalmological evaluation was unremarkable.

Treatment with indomethacin was gradually increased over 2.5 days to $125 \mathrm{mg}$ b.i.d., causing prompt and sustained relief of facial pain and associated symptoms, and the patient was discharged home after 4 days without subjective complaints. Over the course of the next 3 months, indomethacin could be tapered to a maintenance dose of 50 mg q.d., while complete cessation of medication caused immediate exacerbation of symptoms. During the further course, the patient reported only a single re-occurrence of scintillating scotoma of approximately 60 min duration coinciding with a pain exacerbation. Six months after the initial presentation, the patient was finally able to stop indomethacin completely and was without subjective complaints. Repeated neurological examination (follow-up $>12$ months) revealed only mild right-sided residual ptosis, which was far less pronounced than initially. Follow-up cranial MRI (including high-resolution sequences in the area of the cavernous sinus and orbital cavities) performed 7 and 15 months after the initial presentation continued to be unremarkable.

\section{Conclusion}

Clinical features and comprehensive workup, which carefully ruled out underlying secondary etiologies, established a diagnosis of HC based on ICHD-III $\beta$ [1]. In addition to the rather old age of the patient, several other unusual aspects deserve attention: the persistence of mild residual ptosis in the absence of pain is a peculiar feature, which may indicate a dissociation of pain and autonomic symptoms, as suggested by similar observations in other TACs, such as cluster headache [6]. The initially required dose of indomethacin may seem quite high but is well in the range reported in other studies [2]. Another interesting aspect is the observation that indomethacin could be completely tapered off - an unusual feature recognized also in other cases of $\mathrm{HC}$ [7].

The most intriguing feature of our case, however, is the occurrence of transient visual disturbances (i.e., scintillating scotoma) compatible with visual aura accompanying an HC exacerbation. Given the patient's age as well as the negative previous history of both migraine and similar visual symptoms, a random coincidence with isolated visual migraine aura (i.e., with another separate primary headache disorder) seems very unlikely. Instead, we propose a potential (causal) link between $\mathrm{HC}$ and aura - an interpretation which is further corroborated by the fact that aura symptoms also remitted after initiation of indomethacin treatment.

Beyond an observation by Antonaci and Sjaastad [8] of a patient with symptomatic HC and rather unspecific aura symptoms, there have been single reports of $\mathrm{HC}$ associated with aura symptoms, including a single patient with sensory aura and 6 patients with visual aura symptoms [7, 9-11]. In line with what we observed in our patient, these aura symptoms preceded or accompanied episodes of pain exacerbation in all reported cases, both pain and aura symptoms responded completely to treatment with indomethacin, and there was no personal or family history of migraine. While the published cases displayed diverse visual aura features (e.g., flashing lights, white bright flashes, flickering light, and dark spots), our 
observation - to the best of our knowledge - seems to be the first case of HC associated with classic scintillating scotoma.

Beyond the aura-HC link under discussion here, aura symptoms have been shown to occur also in various other primary headache disorders, in particular in TACs: Silberstein et al. [12] reported visual and olfactory aura symptoms in 6 patients with cluster headache, with aura symptoms preceding the attacks, while Vega et al. [13] described a case of aura with visual symptoms and speech disturbances in SUNCT (short-lasting unilateral neuralgiform headache with conjunctival injection and tearing) with good response to lamotrigine.

In summary, these findings highlight the fact that the occurrence of aura symptoms is not necessarily specific to migraine. Taking into account the well-recognized fact that trigeminal autonomic symptoms are also not specific to TACs but may also occur, for example, in migraine, it becomes clear that there is a substantial overlap of clinical symptoms, i.e., phenotypic aspects between different primary headache disorders - a phenomenon which Young et al. [14] addressed with a modular headache concept. In terms of treatment response, this overlap is exemplified, for example, by the observation of a positive treatment effect of the migraine prophylactic agent topiramate also in HC [15].

Coming back to the specific issue of aura symptoms in nonmigraine cases, the fact deserves attention that all reported patients with co-occurrence of aura are from the TAC spectrum, which may argue in favor of shared pathophysiological mechanisms (as suggested, for example, by the close temporal association between headache and aura symptoms and parallel response to treatment) and, potentially, even a common genetic basis.

In summary, our observation reinforces a modular concept of primary headache disorders and demonstrates that aura symptoms may also occur in entities other than migraine, preferentially from the TAC spectrum, suggesting shared pathophysiological mechanisms, which need to be addressed in future functional and genetic studies.

\section{Statement of Ethics}

The patient provided written informed consent for the publication of this case report.

\section{Disclosure Statement}

The authors declare no potential conflicts of interest with respect to the research, authorship, and/or publication of this article.

\section{References}

1 Headache Classification Committee of the International Headache Society (IHS): The international classification of headache disorders, 3rd edition (beta version). Cephalalgia 2013;33:629-808.

-2 Cittadini E, Goadsby PJ: Hemicrania continua: a clinical study of 39 patients with diagnostic implications. Brain 2010;133:1973-1986.

3 Newman LC, Lipton RB, Solomon S: Hemicrania continua: ten new cases and a review of the literature. Neurology 1994;44:2111-2114.

-4 Peres MF, Silberstein SD, Nahmias S, Shechter AL, Youssef I, Rozen TD, et al: Hemicrania continua is not that rare. Neurology 2001;57:948-951.

5 Peres MFP, Siow HC, Rozen TD: Hemicrania continua with aura. Cephalalgia 2002;22:246-248.

-6 Lin H, Dodick DW: Tearing without pain after trigeminal root section for cluster headache. Neurology 2005;65:1650-1651. 
-7 Palmieri A, Mainardi F, Maggioni F, Dainese F, Zanchin G: Hemicrania continua evolving from migraine with aura: clinical evidence of a possible correlation between two forms of primary headache. Cephalalgia 2004;24:1007-1008.

8 Antonaci F, Sjaastad O: Hemicrania continua: a possible symptomatic case, due to mesenchymal tumor. Funct Neurol 1992;7:471-474.

-9 Fantini J, Koscica N, Zorzon M, Belluzzo M, Granato A: Hemicrania continua with visual aura successfully treated with a combination of indomethacin and topiramate. Neurol Sci 2015;36:643-644.

10 Peres MFP, Masruha MR, Young WB: Side-shifting hemicrania continua with aura (migraine with aura with autonomic symptoms responsive to indomethacin?). Cephalalgia 2006;26:917-919.

11 Peres MFP, Siow HC, Rozen TD: Hemicrania continua with aura. Cephalalgia 2002;22:246-248.

$\checkmark 12$ Silberstein SD, Niknam R, Rozen TD, Young WB: Cluster headache with aura. Neurology 2000;54:219_ 221.

13 Vega J, Mauri G, Pascual J: Coexistence of SUNCT and aura? A case report. Cephalalgia 2012;32:940-941.

-14 Young WB, Peres M, Rozen TD: Modular headache theory. Cephalalgia 2001;21:842-849.

15 Matharu MS, Bradbury P, Swash M: Hemicrania continua: side alternation and response to topiramate. Cephalalgia 2006;26:341-344. 